\title{
III. The general determination of the optical constants of a crystal by means of refraction through a prism
}

\section{G.F. Herbert Smith M.A.}

To cite this article: G.F. Herbert Smith M.A. (1906) III. The general determination of the optical constants of a crystal by means of refraction through a prism , Philosophical Magazine Series 6, 12:67, 29-36, DOI: 10.1080/14786440609463508

To link to this article: http://dx.doi.org/10.1080/14786440609463508

曲 Published online: 16 Apr 2009.

Submit your article to this journal 지

ЏII Article views: 2

Q View related articles $\longleftarrow$ 
and sufficient to assume that the electron expands at a certain slow rate $(\$ 4)$.

(2) In order to satisfy the principle of the conservation of energy it is necessary to assume that the expansion is resisted by an internal stress, which in the simplest case is a hydrostatic pressure $(\S 5)$.

(3) In order to explain this pressure in accordance with the spirit of the electron theory, that is, without action at a distance, we must assume that it is due to the action of the æther on the electron $(\$ \$ 6,7)$.

(4) The reaction of the electron on the æether produces a pressure in the ather at the surface of the electron; if this is not exactly balanced by the pressure at infinity the electron expands, presses back the ather and acts like a source in a liquid. Hence electrons gravitate towards each other, as do sources in a liquid $(\$ 8)$.

III. The General Determination of the Optical Constants of a Crystal by means of Refraction through a Prism. By G. F. Herbert Smi'h, M.A., Assistant in the Mineral Department of the British Museum*.

$B^{\mathrm{Y}}$ far the simplest method of obtaining the three principal indices of refraction of a crystalline substance is that of total-reflexion, since observations need be made on only one face or section of the crystal; but this method is necessarily restricted to substances of lower refractive power than the constant medium (usually a hemisphere of dense glass) or the liquid used to obviate the intervention of a film of air. In other cases, however, recourse must be had to refraction through a prism formed by a pair of natural or artificial faces on the crystal, inclined to one another at not too large an angle. If possible, it is arranged that the plane bisecting the angle between these faces is one of symmetry in the crystal, so that the method of minimum deviation and the corresponding simple formula are available. But it is often the case in natural crystals that advantage cannot be taken of this simple method: the crystal may not have suitable natural faces inclined at a small enough angle, and may be so small itself that artificial faces cannot be ground on to it. We propose to show that, even in such cases, it is possible by taking observations sufficiently far round a zone to determine the optical constants.

* Communicated by the Author. The substance of the paper was discussed by him at meetings of the Mineralogical Society, June 19, 1900, and November 17, 1903. 


\section{Mr. G. F. H. Smith on the General Determination}

In his classical report on double refraction which was presented to the British Association at the Cambridge meeting of 1862 , Stokes pointed out* that the velocity of a wave, the normal of which lies in a plane perpendicular to the faces of a prism, and its direction with respect to the faces and, therefore, to the crystallographic axes, can be accurately determined from observations of the angle of incidence and the corresponding angle of deviation, and he gave the formulæ required. Cornu $\dagger$ has more thoroughly discussed the same question, especially in regard to the remarkable tilt of the refracted image with respect to the edge of the prism which is observable in such strongly birefringent substances as calcite and sodium nitrate. $\mathrm{He}$, indeed, measured this tilt in the case of the image given by the extraordinary ray and made use of it for the determination of the extraordinary indices of these substances. In most substances the tilt is not sufficiently perceptible to be used for purposes of measurement.

The formula required in the case of refraction through a prism are as follows:-

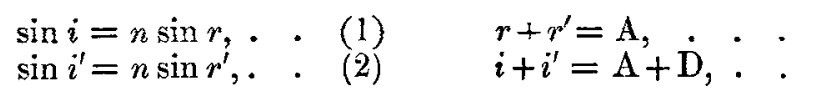

where $i$ and $i^{\prime}$ are the angles which the incident and emergent waves make with the faces of incidence and emergence respectively,

$r$ and $r^{\prime}$ the angles which the refracted wave makes with the same faces,

$n$ is the index of refraction of the wave in the crystalline medium, that of the surrounding medium being assumed to be unity,

A the angle of the prism, and $D$ the angle of deviation.

The above equations reduce to

$$
\tan \left(r-\frac{A}{2}\right)=\tan \frac{A}{2} \tan \left(i-\frac{A+D}{2}\right) \cot \frac{A+D}{2}, .
$$

and we further have

$$
\phi=\frac{r-r^{\prime}}{2}=r-\frac{\mathrm{A}}{2}=\frac{\mathrm{A}}{2}-r^{\prime}, . . .
$$

* Report, p. 273 .

† "De la réfraction à travers un prisme suivant une loi quelconque," Annales scientifiques de l'Ecole Normale Supérieure, ser. 2, 1872, vol. i. pp. 231-272; 1874, vol. iii. pp. 1-46; of. also Liebisch, Physikalische Krystallographie, Leipzig, 1891, pp. 295-8, 376-8, and Walker, 'The Analytical 'Theory of Light,' Cambridge, 1904, pp. 8-15. 
where $\phi$ is the angle which the wave-front in the crystal makes with the plane bisecting the acute angle of the prism.

The angles $i$ and $\mathrm{D}$ can be found by observation with a goniometer, and the values of $n$ and $\phi$ may be calculated from equations (5) and (1) or (2). Thus we can completely determine the velocity (i.e. the inverse of the refractive index, $n$ ) of any plane-wave within the crystal and its orientation with regard to the crystallographic axes.

Suppose we are able to take a series of observations round a zone, $i$. e. through pairs of faces with parallel edges, we should obtain a series of values for the velocity of propagation and the corresponding direction within the crystal, and can plot out curves with the former as ordinates and the latter as abscissa. In the case of a doubly-refracting substance there will be two curves, the nature of which we will now proceed to determine.

Let us take the most general case in which the indicatrix * is an ellipsoid. Let $\mathrm{R}$ be any point on the surface,

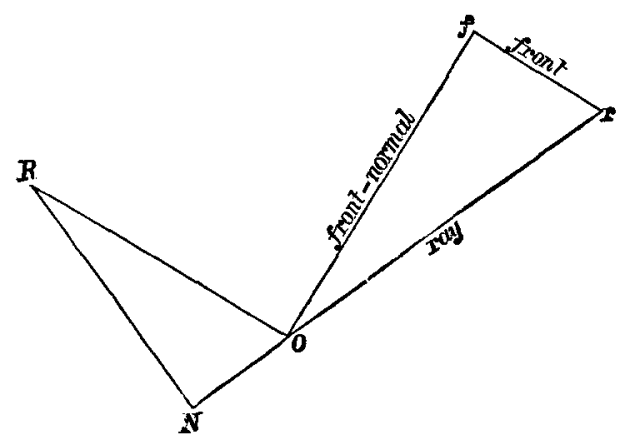

RN the normal at that point, and $O$ the centre of the ellipsoid. Through $\mathrm{O}$ draw $O N$ at right angles to $R N$ and produce it backwards to $r$ so that $\mathrm{O} r=\frac{1}{\mathrm{RN}}$. In the plane $\mathrm{RO} r, \mathrm{O} f$ is drawn at roght angles to $\mathrm{OR}$ to meet $r f$, drawn parallel to $\mathrm{OR}$, in $f$. Then $\mathrm{O} f=\frac{1}{\mathrm{OR}}$. We know that $\mathrm{O} f$ is the normal corresponding to a ray $\mathrm{O} r$, and the plane through $f r$ at right angles to $O f$ is the wave-front, which

* The notation is the same as that employed by Mr. L. Fletcher, F.R.S., "The Optical Indicatrix and the Transmission of Light in Crystals," Mineralogical Magazine, 1891, vol. ix. pp. 278-388; published separately, London, Henry Frowde, 1892. The diagram above is his fig. 12, the block of which was courteously lent to the Author. 


\section{Mr. G. F. H. Smith on the General Determination}

touches the wave-surface at $r$; and further, that for rays with the same direction of wave-normal, the corresponding values of the wave-normal, $p\left(=\frac{1}{n}\right)$, are the inverses of the semi-axes of the perpendicular central-section. Whenever the wave-normal is at right angles to a principal axis, one of the two values obtained is half the inverse of that axis. Hence, if the axes are $\alpha, \beta, \gamma$, supposed to be in ascending order of magnitude, it is obvious from geometrical considerations that the maximum and minimum values in a complete semi-revolution will give us $\gamma$ and $\alpha$. Since each curve has a maximum and a minimum value, the question remains what do the lower maximum and the higher minimum values give; one must clearly be $\beta$.

Let $a^{2} x^{2}+b^{2} y^{2}+c^{2} z^{9}=1$ be the equation to the indicatrix, where $a=\frac{1}{a}, b=\frac{1}{\beta}, c=\frac{1}{\gamma}$; and let $\lambda \mu \nu$ be the direction cosines of the central line parallel to the edge of the prism. Since the wave-normal is always perpendicular to this line, the central section turns round it. Let the equation of this section be $l z+m y+n z=0$.

Then

$$
l \lambda+m \mu+n \nu=0 . \quad . \quad . \quad . \quad . \quad .
$$

Now the semi-axes of the section are given by

$$
\frac{l^{2}}{a^{2}-p^{2}}+\frac{m^{2}}{b^{2}-p^{2}}+\frac{n^{2}}{c^{2}-p^{2}}=0, \quad . \quad .
$$

where $p$ is the inverse of the semi-axis and therefore the value of the corresponding wave-normal.

Further we have $\quad l^{2}+m^{2}+n^{2}=1$.

For critical values of $p, d p=0$. Hence we differentiate the above three equations in the usual way with regard to $l, m$, and $n$ and eliminate the differentials, and arrive at the determinant

$$
\left|\begin{array}{ccc}
\frac{l}{a^{2}-p^{2}} & \frac{m}{b^{2}-p^{2}} & \frac{n}{c^{2}-p^{z}} \\
\lambda & \mu & \nu \\
l & m & n
\end{array}\right|=0
$$

which reduces to

$$
\frac{\lambda}{l}\left(b^{2}-c^{2}\right)\left(a^{2}-p^{2}\right)+\frac{\mu}{m}\left(c^{2}-a^{2}\right)\left(b^{2}-p^{2}\right)+\frac{\nu}{n}\left(a^{2}-b^{2}\right)\left(c^{2}-p^{2}\right)=0 \text {. }
$$


Substituting this value for $p^{2}$ in (8), and remembering the relation in (7), we deduce

$$
\operatorname{lm} n\left\{\frac{l}{\lambda}\left(b^{2}-c^{2}\right)+\frac{m}{\mu}\left(c^{2}-a^{2}\right)+\frac{n}{v}\left(a^{2}-b^{2}\right)\right\}=0,
$$

whence we see that the critical values are

$$
\begin{array}{r}
l=0, m=0, x=0, . . \\
\frac{l}{\lambda}\left(b^{2}-c^{2}\right)+\frac{m}{\mu}\left(c^{2}-a^{2}\right)+\frac{n}{\nu}\left(a^{2}-b^{2}\right)=0 .
\end{array}
$$

If $l=0$, we have from (11) $p=a$; and similarly, if $m$ or $n=0$, we obtain $p=b$ or $c$.

Combining (13) and (7), we get

$$
\begin{aligned}
\bar{\lambda} \frac{l}{\left\{\mu^{2}\left(a^{2}-b^{2}\right)-\nu^{2}\left(c^{2}-a^{2}\right)\right\}} & =\frac{m}{\mu\left\{\nu^{2}\left(b^{2}-c^{2}\right)-\lambda^{2}\left(a^{2}-b^{2}\right)\right\}} \\
& =\frac{n}{\nu\left\{\lambda^{2}\left(c^{2}-a^{2}\right)-\mu^{2}\left(b^{2}-c^{2}\right)\right\}},
\end{aligned}
$$

and substituting in (11) after some reduction we finally ubtain

$$
p^{2}=a^{2} \lambda^{2}+b^{2} \mu^{2}+c^{2} v^{2} .
$$

It will be noticed that this value of $p$ is the inverse of the radius of the ellipsoid which is parallel to the edge of the prism.

We might arrive at the same result in another way. The directions of the axes of any central-section, whose equation is $l x+m y+n z=0$, is its intersection with the cone

$$
\frac{l}{x}\left(b^{2}-c^{2}\right)+\frac{m}{y}\left(c^{2}-a^{2}\right)+\frac{n}{z}\left(a^{2}-b^{2}\right)=0 .
$$

Comparing these equations with (13) and (7) we see at once that the fourth critical value is the inverse of the semidiameter parallel to the edge of the prism.

Let us consider which of the critical values are maxima or minima. We must differentiate equations (7), (8), and (9) twice, regarding one of the variables, let us say $m$, as independent. We have

$$
\begin{aligned}
\frac{d l}{d m} & =\frac{m v-n \mu}{n \lambda-l v}, & \frac{d n}{d m} & =\frac{l \mu-m \lambda}{n \lambda-l v}, \\
\frac{d^{2} l}{d m^{2}} & =\frac{\nu}{(n \lambda-l v)^{3}}, & \frac{d^{2} n}{d m^{2}} & =\frac{-\lambda}{(n \lambda-l \nu)^{3}} .
\end{aligned}
$$

Plit. Mag. S. 6. Vol. 12. No. 67. July 1906. 
34 Mr. G. F. H. Sinith on the General Determination.

Arrange (11) thus

$$
p^{4}-p^{2}\left\{l^{2}\left(b^{2}+c^{2}\right)+m^{2}\left(e^{2}+a^{2}\right)+n^{2}\left(a^{2}+b^{2}\right)\right\}+l^{2} b^{2} c^{2}+m^{2} c^{2} a^{2}+n^{2} a^{2} b^{2}=0
$$

and differentiate twice, remembering that $\frac{d p}{d m}$ vanishes for the critical values.

$$
\begin{gathered}
p \frac{d^{2} p}{d m^{2}}\left\{2 p^{2}-l^{2}\left(b^{2}+c^{2}\right)-m^{2}\left(c^{2}+a^{2}\right)-n^{2}\left(a^{2}+b^{2}\right)\right\}-p^{2}\left[( b ^ { 2 } + c ^ { 2 } ) \left\{l \frac{d^{2} l}{d m^{2}}\right.\right. \\
\left.\left.+\left(\frac{d l}{d m}\right)^{2}\right\}+\left(c^{2}+a^{2}\right)+\left(a^{2}+b^{2}\right)\left\{n \frac{d^{2} n}{d m^{2}}+\left(\frac{d n}{d m}\right)^{2}\right\}\right] \\
+b^{2} c^{2}\left\{l \frac{d^{2} l}{d m^{2}}+\left(\frac{d l}{d m}\right)^{2}\right\}+c^{2} a^{2}+a^{2} b^{2}\left\{n \frac{d^{2} n}{d m^{2}}+\left(\frac{d n}{d m}\right)^{2}\right\}=0 .
\end{gathered}
$$

Putting $p=b$ and $m=0$, we have

$$
b \frac{d^{2} p}{d m^{2}} \cdot\left\{2 b^{2}-l^{2}\left(b^{2}+c^{2}\right)-n^{2}\left(a^{2}+b^{2}\right)\right\}=\left(a^{2}-b^{2}\right)\left(b^{2}-c^{2}\right) .
$$

Suppose $b$ is greater than $a$ and $c$. The term on the right is negative, and the expression within the bracket on the left, which can be arranged thus $l^{2}\left(b^{2}-c^{2}\right)+n^{2}\left(b^{2}-a^{2}\right)$, is positive. Hence $\frac{d^{2} p}{d m^{2}}$ is negative, and, as indeed was otherwise obvious, the greatest value of $p$ is a maximum value of the corresponding curve. Similarly the least value is a minimum value.

If now $b$ is the mean value, the term on the right is positive. Again, since $m=0, l \lambda+n \nu=0$, and, therefore,

$$
l^{2}=\frac{\nu^{2}}{y^{2}+\lambda^{2}}, \quad n^{2}=\frac{\lambda^{2}}{v^{2}+\lambda^{2}} .
$$

Substituting these values in the expression within the bracket we have $\left\{b^{2}-\left(a^{2} \lambda^{2}+b^{2} \mu^{2}+c^{2} y^{2}\right)\right\} /\left(v^{2}+\lambda^{2}\right)$. But $a^{2} \lambda^{2}+b^{2} \mu^{2}+c^{2} \nu^{2}$ is the square of the semi-diameter parallel to the edge of the prism. Hence, if the diameter be greater than the mean axis of the ellipsoid, the value corresponding to the latter is a minimum value; if less, a maximum value. Now at any point lying on the circular sections, the semidiameter is equal to the mean semi-axis. If, therefore, the direction parallel to the edge of the prism lies towards the greatest axis of the ellipsoid, the corresponding value is a maximum; but, if towards the least, it is a minimum ; if it lies on either of the circular sections, the two curves touch.

The two intermediate values may be discriminated by determining the angles of extinction with regard to the elge 
of the prism. The plane of polarization of any wave is at right angles to the normal at the corresponding point on the indicatrix. The angles of extinction will be in the case of the principal axes $\cos ^{-1} \lambda, \cos ^{-1} \mu, \cos ^{-1} \nu$, and for the fourth value $\cos ^{-1} \frac{a^{2} \lambda^{2}+b^{2} \mu^{2}+c^{2} \nu^{2}}{\sqrt{a^{4} \lambda^{2}+b^{4} \mu^{2}+c^{4} \nu^{2}}}$.

The values for the three principal axes must satisfy the condition $\quad \lambda^{2}+\mu^{2}+\nu^{2}=1$.

It may be noted that the planes of polarization for the two rays with parallel wave-fronts are in general not strictly at right angles, since the normals at the extremities of the axes of the central-section to which they are perpendicular are not themselves in general at right angles.

Another point that is worthy of notice is that the two curves are in general not symmetrical about the critical positions. The abscissæe, as we have remarked above, represent the angles which the wave-normal makes with some fixed direction; or, in other words, the angles which the central section revolving round the diameter parallel to the edge of the prism makes with some fixed plane. Supposing the upper curve contains the greatest and the mean of the principal indices, the angle between the corresponding positions of the central section is

$$
\cos ^{-1} \frac{\lambda \mu}{\sqrt{\left(\mu^{2}+\nu^{2}\right)\left(\nu^{2}+\lambda^{2}\right)}},
$$

which in general is not a right angle; on the lower curve the angle is

$$
\cos ^{-1} \frac{\lambda \mu\left(a^{2}-b^{2}\right)}{\sqrt{\left(\lambda^{2}+\mu^{2}\right)\left\{\left(b^{2}-c^{2}\right)^{2} \mu^{2} \nu^{2}+\left(c^{2}-a^{2}\right)^{2} \nu^{2} \lambda^{2}+\left(a^{2}-b^{2}\right)^{2} \lambda^{2} \mu^{2}\right\}}} \cdot
$$

If the crystal be uniaxial the upper curve becomes a straight line, and the lower curve, which is still undulating, is now symmetrical, since $a=b$ and the angle between the critical positions is a right angle.

Although the tilt of the refracted image, as was mentioned above, is in general not sufficiently perceptible for purposes of measurement, it may be indirectly of service, because its existence signifies that the corresponding direction in the crystal is not near one of the critical positions and, therefore, no observations need be taken. The range inside the crystal corresponding to a particular prism may be increased if the observations are made on an inverted goniometer in some liquid medium. In an instrument of this kind which is in the British Museum light passes into and out of the liquid by means of double-ended glass-prisms. As the angle of incidence D 2 
diminishes, it is clear that a point will be reached beyond which it will not be. possible to measure it directly because the telescope would come into contact with the collimator. Indirect, though perfectly accurate, determinations may be made by clamping the erystal-holder rigidly to the graduated circle and noting the reading in any position when the telescope is directly opposite to the collimator. If a certain direct reading $\theta$ has been found to correspond to an angle of incidence $i$, then a reading $\theta \pm \omega$ (depending on whether the angle of incidence diminishes or increases with the reading) corresponds to an angle of incidence $i-\omega$. This is, indeed, the most convenient way of determining the angles of jncidence.

Obviously much labour may be avoided if previously the orientation of the indicatrix has been determined either from the crystallographical symmetry where possible, or from observations under the polariscope.

IV. Ellipsoidal IIarmonics, Eolotropic and Isotropic. By R. Hararkaves, M.A.*

T $T$ is the first object of the present paper to show that 1 Lamés functions are applicable to the æolotropic form of the equation of Laplace, with some modification in the meaning of the constants used, and practically no change in the form of the results. It is also shown that in rolotropic and in isotropic work, the parametral equations required to determine Lamé's functions in every case involve a single constant of the ellipsoid. This point is obscured in Heine's treatment of the functions by the use of the unsymmetrical notation in which squares of semi-axes are represented by $\lambda^{2}, \lambda^{2}-a^{2}, \lambda^{2}-b^{2}$. If $a^{2}+\lambda, b^{2}+\lambda, c^{2}+\lambda$ are used there is exact correspondence between the classification of curvilinear and Cartesian forms, i.e. distinctions depend on the presence or absence of the radicals $\sqrt{\lambda+a^{2}}, \ldots$ in the one case, of the linear factors $x y z$ in the other. This follows from the connexion between curvilinear parameters $\lambda \mu \nu$ and Cartesians, which for isotropy is given by

$f \equiv u_{\theta}-1 \equiv \frac{x^{2}}{a^{2}+\theta}+\frac{y^{2}}{b^{2}+\theta}+\frac{z}{c^{2}+\theta}-1 \equiv \frac{\overline{\lambda-\theta} \overline{\mu-\theta} \overline{\nu-\theta}}{\overline{a^{2}+\theta} \overline{b^{2}+\theta} \overline{c^{2}+\theta}}$.

If $\mathrm{R}_{n}$ is used for a product $f_{1} \ldots f_{n}$ in which the subscripts correspond to different values $\theta_{1}, \ldots \theta_{n}$, there are normal

* Communicated by the Autbor. 